\title{
Nolina exCelsa (NolinaCEAe) UnA eSPECIE NUEVA Del estado de OAXaca, MÉxico
}

\author{
Abisaí García-Mendoza ${ }^{1,3}$, Eloy Solano ${ }^{2}$ y Miguel Rivera-Lugo ${ }^{2}$ \\ 'Jardín Botánico, Instituto de Biología, Universidad Nacional Autónoma de México \\ ${ }^{2}$ Unidad de Investigación en Sistemática Vegetal y Suelo, Herbario FEZA, Facultad de Estudios Superiores Zaragoza, \\ Universidad Nacional Autónoma de México \\ ${ }^{3}$ Autor para la correspondencia: abisai@ibiologia.unam.mx
}

\begin{abstract}
Resumen: Se describe e ilustra Nolina excelsa, especie nueva del estado de Oaxaca. Esta especie se compara con N. parviflora, de la cual difiere en la altura de los individuos, la longitud y número de hojas por roseta; los tépalos con una línea media longitudinal de color morado; el tamaño del fruto y la forma, tamaño y ornamentación de las semillas.

Palabras clave: Asparagaceae, Liliaceae, Nolina parviflora, Ruscaceae.
\end{abstract}

\begin{abstract}
Nolina excelsa, a new species from Oaxaca, Mexico, is described and illustrated. This species is compared with N. parviflora, from which it differs in height of individuals, length and number of leaves per rosette; tepals with a longitudinal medium purple line; the size of the fruit, and, shape, size and ornamentation of the seeds.
\end{abstract}

Key words: Asparagaceae, Liliaceae, Nolina parviflora, Ruscaceae.

$N$ olina Michx. (1803) es un género americano con 21 a 30 especies (Trelease, 1911; Hess, 2002; Hochstätter, 2010) que se distribuyen desde el sur de los Estados Unidos de América hasta México, con su límite de distribución meridional en el estado de Oaxaca. La primera revisión taxonómica del género fue realizada por Trelease (1911) quien incluyó 21 especies, agrupadas en cuatro secciones [Graminifoliae (=Nolina), Erumpentens, Microcarpae y Arborescentes], delimitadas con base en el hábito, las características de las hojas, el tipo de inflorescencias y los frutos. El género ha sido poco estudiado desde el punto de vista taxonómico; entre los trabajos recientes están el de Dice (1988), quien abordó la sección Arborescentes de Nolina para los Estados Unidos de América, el de Hess y Dice (1995) quienes publicaron una especie nueva de California y el de Hess (2002) quien realizó el tratamiento taxonómico del género para la Flora de Norteamérica, describiendo 14 especies. Para México, Trelease (1911), Standley (1920) y Hochstätter (2010) consideraron 17 especies, ubicadas en las cuatro secciones mencionadas, diez de ellas endémicas del país. Por su parte, Espejo-Serna y López-Ferrari (1996) registraron para el país 20 especies y dos variedades.
Nolina y sus géneros relacionados Beaucarnea, Calibanus y Dasylirion, han sido considerados dentro de las familias Liliaceae (Bentham y Hooker, 1883; Engler, 1888; Standley, 1920; Krause, 1930; McVaugh, 1989), Agavaceae (Hutchinson, 1934; Cronquist, 1981; Verhoek y Hess, 2002), Dracaenaceae (Takhtajan, 1986; Brummitt, 1992) o Nolinaceae (Dahlgren et al., 1985, Bogler, 1998; Walker, 2001). La familia Nolinaceae fue propuesta por Nakai (1943) y está constituida por los géneros anteriormente mencionados. Como resultado de algunos estudios filogenéticos publicados y con base en evidencias morfológicas y moleculares, Nolina y sus géneros emparentados han sido ubicados también en Convallariaceae (APG, 1998), Ruscaceae (APG II, 2003; Judd et al., 2008) o como subfamilia Nolinoideae dentro de Asparagaceae (APG III, 2009). Sin embargo, esta última propuesta no considera a Asparagaceae como una familia monofilética. Por lo tanto, en este trabajo preferimos seguir ubicando a Nolina como parte de la familia Nolinaceae hasta no tener evidencias morfológicas y moleculares más concretas.

Durante la preparación del tratado taxonómico de Nolina (Nolinaceae) para la Flora del Valle de Tehuacán-Cuicatlán, 
se revisaron algunos especímenes con características morfológicas diferentes a las especies descritas con anterioridad, por lo que proponemos como especie nueva a:

Nolina excelsa García-Mend. et E. Solano sp. nov. (Figura 1). Herba perennis, arborescens, $8-13 \mathrm{~m}$ alta, $40-50 \mathrm{~cm}$ diametro, 8-16 ramis. Folia circa 100 per rosulam; laminae (60-) $70-85 \mathrm{~cm}$ longae, lineares, rectae, glaucae. Flores velut unisexuales, fungentes, albidae; linea purpurea in parte media tepalorum. Fructus 7-8.5 mm longi. Semina 4-4.2 mm longa, globosa, brunnea.

TIPO: México, Oaxaca, ladera oeste del Cerro la Calavera, 7 km en línea recta al este de San Juan Bautista Suchitepec, municipio de San Juan Bautista Suchitepec, distrito Huajuapan, alt. 2,403 m, bosque de Quercus con elementos de matorral xerófilo, plantas femeninas y masculinas, 10 marzo 2011, R. Redonda-Martínez, R. Medina-Lemos, E.M. Martínez-Salas y L.E. de la Cruz-López 481 (Holotipo: MEXU-4 partes; Isotipo: FEZA-4 partes).

Plantas perennes, dioicas, arborescentes 8-13 m de alto, 40$50 \mathrm{~cm}$ de diámetro, base ensanchada, corteza externa fisurada, grisácea a negruzca con ornamentación rectangular; generalmente con 8 a 16 ramas. Hojas aproximadamente 100 por roseta, las secas prontamente caedizas, alternas, espiraladamente dispuestas, rectas aún con la edad; base 5-6 $\mathrm{cm}$ de largo, 4.5-5.5 cm de ancho, deltoide, engrosada, blanquecina, láminas (60-) 70-85 cm de largo, $1.4-2.1 \mathrm{~cm}$ de ancho, lineares, cartáceas, glaucas, ápice largamente atenuado, margen denticulado, los dientecillos disminuyen en tamaño y se separan más hacia el ápice, el tercio distal entero y hialino, haz y envés con superficie estriada. Inflorescencia paniculada, erecta, laxa, 1.2-1.6 (-2.5) $\mathrm{m}$ de largo, pedúnculo $25-35 \mathrm{~cm}$ de largo, $4-4.5 \mathrm{~cm}$ de diámetro en la base, brácteas basales $35-40 \mathrm{~cm}$ de largo, 3-6 cm de ancho, las de la parte media $12-18 \mathrm{~cm}$ de largo, $2.5-3.5 \mathrm{~cm}$ de ancho, las apicales de 8-10 cm de largo, 2-2.5 cm de ancho, lanceoladas, adpresas, papiráceas, disminuyendo en longitud hacia el ápice, margen entero o algo denticulado, blanquecinas, escariosas; porción fértil 95-100 (-115) cm de largo; ramas primarias aproximadamente $100,12-14 \mathrm{~cm}$ de largo, generalmente con 2 (3-4) flores por nudo, fructificando sólo una en el caso de las inflorescencias femeninas; bractéolas 3-9 $\mathrm{mm}$ de largo, 2-5 $\mathrm{mm}$ de ancho, ovadas a deltoides, margen fimbriado, papiráceas, blanquecinas. Flores unisexuales, las estaminadas con pistilodios reducidos, las pistiladas con estaminodios pequeños; pedicelos 3.5-4 mm de largo, en fructificación 9-12 mm de largo, articulados en el tercio basal; tépalos 6, dispuestos en 2 series de 3, 3-4 mm de largo, 1.8$2 \mathrm{~mm}$ de ancho, libres, casi iguales, elípticos a obovados, ápice apiculado, papilado, en flores masculinas reflexos, en las femeninas cóncavos, blanquecinos, con una coloración morada en la línea media del tépalo, margen hialino; estambres 6 , libres, filamentos 2.5-3 mm de largo, más cortos que los tépalos, anteras ca. $1.7 \mathrm{~mm}$ de largo; ovario súpero, ca. $2 \mathrm{~mm}$ de largo, astilar, 3-lobado, lóbulos redondeados, estigma ca. $1 \mathrm{~mm}$ de largo, 3-lobado. Fruto una cápsula trisectada, 7-8.5 mm de largo, 7-9 mm de diámetro en sección transversal, sectos elipsoidales, redondeados distalmente, inflados, pericarpio delgado, los lóculos con una o a veces con dos semillas. Semillas 3.5-4.2 mm de largo, 3-3.8 mm de ancho, subglobosas, muriculadas, pardas oscuras.

Distribución y ecología. Nolina excelsa se conoce únicamente de una región reducida del estado de Oaxaca, situada al este del distrito de Huajuapan y al norte de Teposcolula, entre 2,300 y 2,700 m de altitud, en laderas con pendientes pronunciadas, sobre leptosoles y regosoles delgados y pedregosos. Se desarrolla en el ecotono entre bosque de Quercus y matorral xerófilo con Agave convallis, A. nuusaviorum, A. potatorum, Arctostaphylos pungens, Dasylirion serratifolium, Furcraea longaeva, Hechtia glomerata, Juniperus flaccida, Mimosa lacerata, Opuntia sp., Pittocaulon praecox, Quercus castanea, Q. laeta y Yucca mixtecana.

Fenología: Florece de febrero a abril, fructifica de abril a julio.

Nombre común y usos. Se le conoce con el nombre de sotol. Las hojas se utilizan para construir techos de casas, y con los tallos secos se hacen tablas sobre las que se raspan las hojas de Agave convallis (maguey escobeta) para obtener fibras.

Etimología. El epíteto específico hace referencia a la altura de los individuos.

Por su hábito arborescente, hojas de 1.5-4 $\mathrm{cm}$ de ancho con el margen denticulado, inflorescencia paniculada, amplia, robusta, brácteas del pedúnculo papiráceas, bractéolas fimbriado-laceradas y frutos grandes, Nolina excelsa se ubica en la sección Arborescentes, propuesta por Trelease (1911). Por su morfología se relaciona con $N$. parviflora (Kunth) Hemsl., de la que se diferencia por su mayor altura, hojas glaucas más cortas y en menor número; tépalos blanquecinos con una línea morada en la parte media, frutos de 7-8.5 $\mathrm{mm}$ de largo por 7-9 $\mathrm{mm}$ de diámetro y semillas pardo oscuras con ornamentación de la superficie muriculada. Además, ambas especies florecen y fructifican en épocas diferentes (Cuadro 1). La nueva especie aquí descrita es endémica de las montañas de la Mixteca Alta, en los distritos de Huajuapan y Teposcolula, aunque es probable su presencia en áreas del sur del estado de Puebla, en el municipio de Caltepec. Nolina parviflora también es endémica de México, distribuyéndose desde Durango y Zacatecas, hasta Oaxaca. La 


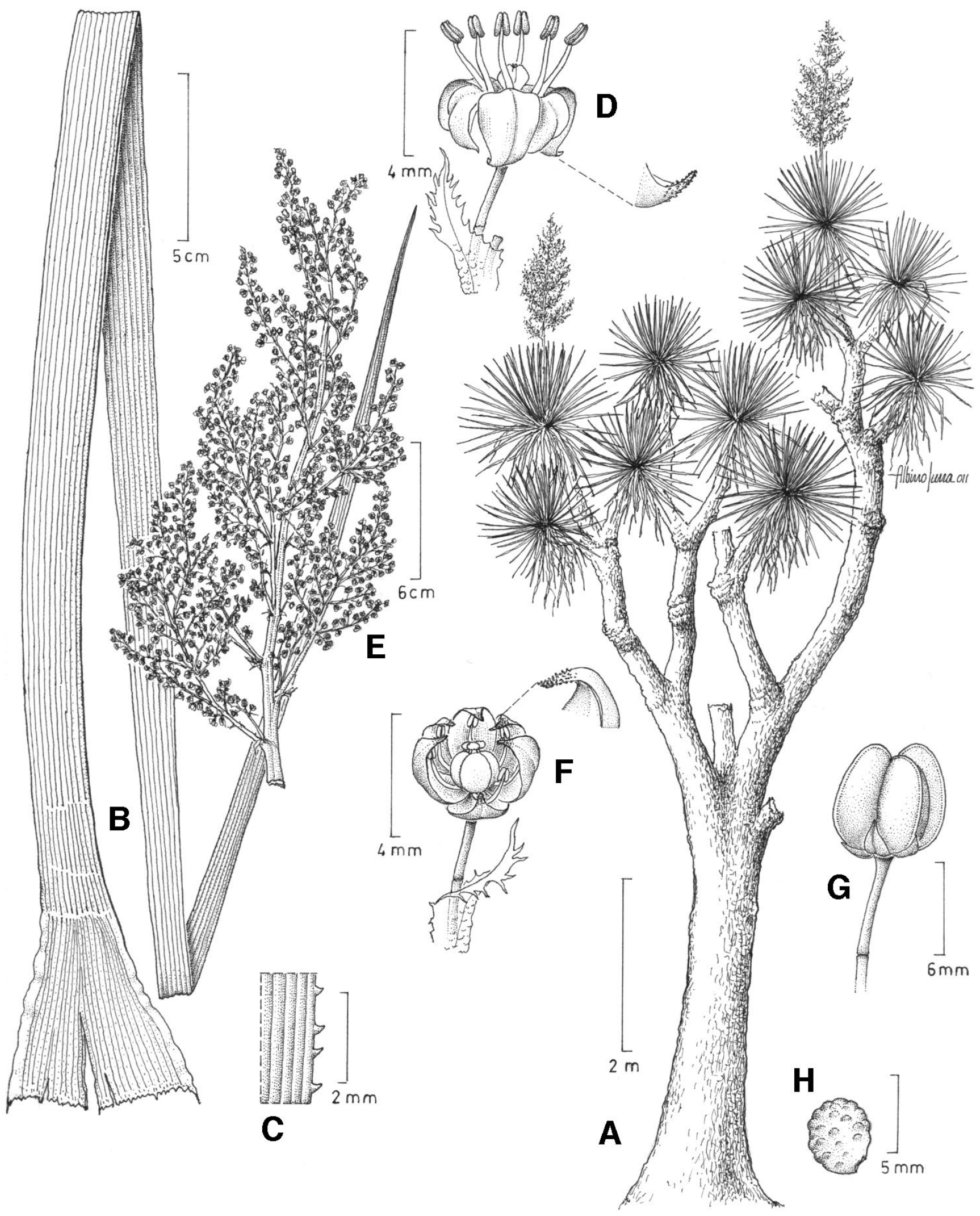

Figura 1. Nolina excelsa. A. Hábito. B. Hoja. C. Detalle del margen de la hoja. D. Flor masculina. E. Rama de la inflorescencia. F. Flor femenina. G. Fruto. H. Semilla. 
Cuadro 1. Comparación de algunas características morfológicas de Nolina excelsa y N. parviflora

\begin{tabular}{|c|c|c|}
\hline Carácter & Nolina excelsa & Nolina parviflora \\
\hline Altura de la planta & 8-13 m & $4-5 \mathrm{~m}$ \\
\hline Hojas por roseta & ca. 100 & $150-200$ \\
\hline Largo de las hojas & $70-80 \mathrm{~cm}$ & $100-150 \mathrm{~cm}$ \\
\hline Color de las hojas & glaucas & $\begin{array}{c}\text { verdes a } \\
\text { verde-amarillentas }\end{array}$ \\
\hline Hojas secas & $\begin{array}{l}\text { prontamente } \\
\text { caedizas }\end{array}$ & persistentes \\
\hline $\begin{array}{l}\text { Postura de las hojas } \\
\text { Coloración de } \\
\text { los tépalos }\end{array}$ & $\begin{array}{c}\text { rectas } \\
\text { blanquecinos con } \\
\text { una línea morada en } \\
\text { la parte media }\end{array}$ & $\begin{array}{l}\text { recurvadas o rectas } \\
\text { blanquecinos con } \\
\text { una línea parda a } \\
\text { verdosa en la } \\
\text { parte media }\end{array}$ \\
\hline $\begin{array}{l}\text { Largo y diámetro } \\
\text { del fruto }\end{array}$ & $7.0-8.5 \times 7-9 \mathrm{~mm}$ & $8-10 \times 10-14 \mathrm{~mm}$ \\
\hline $\begin{array}{l}\text { Largo y diámetro } \\
\text { de la semilla }\end{array}$ & $3.5-4.2 \times 3-3.8 \mathrm{~mm}$ & $3.5-4 \times 2.5-3.5 \mathrm{~mm}$ \\
\hline Forma de la semilla & subglobosa & elipsoidal a ovoide \\
\hline $\begin{array}{l}\text { Ornamentación de } \\
\text { la semilla }\end{array}$ & muriculada & $\begin{array}{l}\text { reticulada o } \\
\text { punctiforme }\end{array}$ \\
\hline $\begin{array}{l}\text { Floración y } \\
\text { fructificación }\end{array}$ & febrero a julio & julio a febrero \\
\hline
\end{tabular}

especie aquí descrita es la más alta de las nolinas mexicanas. De acuerdo con Ricker y Hernández (2010), sólo cuatro especies tienen alturas mayores a los 5 metros: Nolina beldingii Brandegee (7 m), N. matapensis Wiggins ( $6 \mathrm{~m}), N$. nelsonii Rose $(8 \mathrm{~m})$ y $N$. parviflora $(5 \mathrm{~m})$. Nolina excelsa se desarrolla en la Mixteca Alta, área con numerosos endemismos, entre ellos, los géneros Ainea (Iridaceae) y Paneroa (Asteraceae).

Ejemplares adicionales examinados: México, Oaxaca, 1 $\mathrm{km}$ al norte del Portezuelo Mole, $10 \mathrm{~km}$ al noroeste de El Rodeo, distrito Coixtlahuaca, alt. 2,500 m, matorral xerófilo, 9 jul 1986, A. García Mendoza et al. 2500 (MEXU, MO); Cerro Pericón, 24 km N de Tamazulapan, 6 km N de San Pedro Nopala, distrito Teposcolula, alt. 2,360 m, matorral rosetófilo, 11 abr 1987, A. García Mendoza et al. 3075 (MEXU, MO); Cerro Pericón, 6 km N de San Pedro Nopala, camino a Yosocuno, alt. 2,700 m, bosque de Quercus, 16 abr 1990, A. García Mendoza 4578 (MEXU); Portezuelo del Frijolillo, falda norte del Cerro Flecha, $5 \mathrm{~km}$ E de San Pedro Nopala, alt. 2,490 m, matorral xerófilo, 22 mar 1996, A. García Mendoza y L. de la Rosa 6167 (MEXU); La cumbre Hijadero, $6 \mathrm{~km}$ O de San Pedro Nopala, alt. 2,510 m, matorral xerófilo. 24 abr 2010, E. Solano Camacho et al. 3468 (FEZA, MEXU); La Cumbre Hijadero, 6 km O de San Pedro Nopala, alt. 2,510 m, matorral xerófilo, 24 abr 2010, E. Solano Camacho et al. 3469 (FEZA, MEXU); Llano del
Frijolillo, 8 km O de San Pedro Nopala, alt. 2,517 m, matorral xerófilo, 27 abr 2010, E. Solano Camacho et al. 3470 (FEZA, MEXU); 6 km SE de San Pedro Nopala, alt. 2,640 m, matorral xerófilo, 25 mar 2011, E. Solano Camacho et al. 3550 (FEZA, MEXU); 6 km SE de San Pedro Nopala, alt. 2,640 m, matorral xerófilo, 25 mar 2011, E. Solano Camacho et al. 3551 (FEZA, MEXU).

\section{Agradecimientos}

Rosalinda Medina Lemos y Fernando Chiang revisaron el escrito final e hicieron importantes observaciones que mejoraron sustancialmente el mismo. Fernando Chiang preparó la diagnosis en latín. La ilustración es obra de Albino Luna. El segundo autor agradece el apoyo financiero de PAPIITUNAM convenio IN225210-3.

\section{Literatura citada}

APG [Angiosperm Phylogeny Group]. 1998. An ordinal classification for the families of flowering plants. Annals of the Missouri Botanical Garden 85:531-553.

APG II. 2003. An update of the Angiosperm Phylogeny Group classification for the orders and families of flowering plants: APG II. Botanical Journal of the Linnean Society 141:399-436.

APG III. 2009. An update of the Angiosperm Phylogeny Group classification for the orders and families of flowering plants: APG III. Botanical Journal of the Linnean Society 161:105-121.

Bentham G. y Hooker J.D. 1883. Genera Plantarum III. Reeve and Co., Londres.

Bogler D. 1998. Nolinaceae. En: Kubitzki K. Ed. The Families and Genera of Vascular Plants. Volume III: Flowering Plants Monocotiledons: Lilianae (except Orchidiaceae), pp. 392-396, Springer-Verlag, Berlín.

Brummitt R.K. 1992. Vascular Plant Families and Genera. Royal Botanic Gardens, Kew.

Cronquist A. 1981. An Integrated System of Classification of Flowering Plants. Columbia University Press, Nueva York.

Dahlgren R.M., Clifford H.T y Yeo P.F. 1985. The Families of the Monocotyledons: Structure, Evolution and Taxonomy. Springer-Verlag, Nueva York.

Dice J.C. 1988. Systematic Studies in the Nolina bigelovii-N. parryi (Nolinaceae) Complex. Tesis de Maestría, San Diego State University, San Diego. 203 pp.

Engler A. 1888. Liliaceae. En: Engler A. y Prantl K. Eds. Die Natürlichen Pflanzenfamilien T2, Ab5, pp. 10-91, Verlag, Leipzig.

Espejo-Serna A. y López-Ferrari A.R. 1996. Las Monocotiledóneas Mexicanas. Una Sinopsis Florística. 1. Lista de Referencia. Parte VI. Dioscoreaceae a Nolinaceae. Consejo Nacional de la Flora de México-Universidad Nacional Autónoma Metropolitana Iztapalapa-Comisión Nacional para el Conocimiento y Uso de la Biodiversidad, México, D.F.

Hess W.J. 2002. Nolina Michaux. En: Flora of North America Editorial Committee. Eds. Flora of North America. Volume 26 Magnoliophyta: Liliidae: Liliales and Orchidales, pp. 415-421, Oxford University Press, Nueva York.

Hess W.J. y Dice J.C. 1995. Nolina cismontana (Nolinaceae), a New Species Name for an Old Taxon. Novon 5:162-164. 
Hochstätter F. 2010. Il Genere/The genus Nolina (Nolinaceae). Gli Speciali di Piante Grasse. Associazione Italiana Amatori delle Piante Succulente, Bolonia.

Hutchinson J. 1934. The Families of Flowering Plants. Vol II. Monocotyledons. Macmillan, Londres.

Judd W.S., Campbell C.S., Kellogg E.A., Stevens P.F. y Donoghue M.J. 2008. Plant Systematics: A Phylogenetic Approach. Sinauer Associates, Sunderland.

Krause K. 1930. Liliaceae. En: Engler A. y Prantl K. Eds. Die Natürlichen Pflanzenfamilien Band 15a, pp. 227-386, Wilhelm Engelmann, Leipzig.

McVaugh R. 1989. Nolina Michaux. En: Anderson W.R. Ed. Flora Novo-Galiciana: Bromeliaceae to Dioscoreaceae. Vol 15, pp. 240-244, The University of Michigan Herbarium, Ann Arbor.

Michaux A. 1803. Flora Boreali Americana. Volume 1. Caroli Capprelet, Paris.

Nakai T. 1943. Ordines, Familiae, Tribi, Genera, Sectiones... novis edita. Appendix. Quaestiones characterium naturalium plan- tarum. Chosakuronbun Mokuroku 226.

Ricker M. y Hernández H.M. 2010. Tree and tree-like species of Mexico: gymnosperms, monocotyledons, and tree ferns. Revista Mexicana de Biodiversidad 81:27-38.

Standley P.C. 1920. Trees and shrubs of Mexico. Contributions from the United States National Herbarium 23:94-97.

Takhtajan, A. 1986. Floristic Regions of the World. University of California Press, Berkeley.

Trelease W. 1911. The desert group Nolineae. Proceedings of the American Philosophical Society 50:404-443.

Verhoek S. y Hess W.J. 2002. Agavaceae Dumortier. En: Flora of North America Editorial Committee. Eds. Flora of North America. Volume 26 Magnoliophyta: Liliidae: Liliales and Orchidales, pp. 413-465, Oxford University Press, Nueva York.

Walker C.C. 2001. Nolina. En: Eggli U. Ed. Illustrated Handbook of Succulent Plants: Monocotyledons, pp. 291-292, SpringerVerlag, Berlín.

Recibido: 4 de octubre de 2011

Aceptado: 24 de enero de 2012 